\title{
光谱法研究蛋白质与表面活性剂的相互作用
}

\author{
吴 丹 徐桂英* \\ (山东大学胶体与界面化学教育部重点实验室, 济南 250100)
}

\begin{abstract}
摘要 结合本课题组的工作, 较系统地总结了近年来有关紫外吸收光谱、苂光光谱、圆二色光谱和电子自旋共振 光谱技术在蛋白质-表面活性剂混合体系研究中的应用. 大量研究表明, 借助于光谱技术不仅可以研究蛋白质结 构与功能的关系, 而且可以探讨蛋白质与表面活性剂的作用机理.
\end{abstract}

关键词：蛋白质，表面活性剂，紫外吸收光谱，苂光光谱，圆二色光谱，电子自旋共振光谱，相互作用 中图分类号: O648, O647

\section{Study on Protein-Surfactant Interaction by Spectroscopic Methods}

\author{
WU, Dan XU, Gui-Ying* \\ (Key Laboratory of Colloid \& Interface Chemistry, Ministry of Education, Shandong University, Jinan 250100, P. R. China)
}

\begin{abstract}
Our results and the applications of spectroscopic methods in studying protein-surfactant system are systematically summarized. Those methods include UV absorption spectroscopy, fluorescence spectroscopy, circular dichroism and electron spin resonance. Many studies indicate that spectroscopic methods can be used to not only study the relationship between structure and function of protein but also probe the interaction mechanism of protein and surfactant.
\end{abstract}

Keywords: Protein, Surfactant, UV absorption spectroscopy, Fluorescence spectroscopy, Circular dichroism, Electron spin resonance, Interaction

蛋白质-表面活性剂混合体系在药物、化妆品、 生物及食品等领域中具有广泛而重要的应用. 例如, 人发和羊毛就是经常暴露于表面活性剂中的两种 蛋白质底物;一些食品配方中常常同时含有蛋白质 和表面活性剂. 因此蛋白质和表面活性剂相互作用 的研究是多年来人们一直十分感兴趣的课题 ${ }^{[-7]}$. 蛋 白质和表面活性剂的相互作用与聚合物和表面活 性剂的相互作用有许多相似之处. 但从分子结构考 虑, 蛋白质是一类具有特殊结构的两性聚电解质, 存在等电点, 随着溶液 $\mathrm{pH}$ 的不同, 分子的荷电性质 发生很大变化. 尤其是蛋白质具有二级和三级结构, 明显不同于其它类型的聚电解质, 因此, 蛋白质与 表面活性剂的相互作用具有许多独特之处, 而且更
为复杂.

研究表面活性剂与蛋白质相互作用的方法很 多 ${ }^{[-23]}$, 最早使用的有表面张力、电导、离子选择性电 极和粘度法等. 目前研究最多、应用最广的有光散 射、光谱法以及新发展起来的小角 X-射线散射、小 角中子散射、Brewster 角显微镜等方法. 我们曾在蛋 白质和小分子物质的相互作用方面开展了一些工 作 ${ }^{[24-30]}$, 采用表面张力和光谱法等手段研究过蛋白 质与表面活性剂的相互作用, 并探讨了混合体系的 铺展行为和泡沫性能等. 本文结合课题组的工作, 着重阐述了光谱法中的紫外吸收光谱、苂光光谱及 近年来兴起的圆二色光谱、电子自旋共振光谱在蛋 白质-表面活性剂相互作用研究中的应用.

Received: July 8, 2005; Revised:August 22, 2005. "Correspondent, E-mail:xuguiying @ sdu.edu.cn; Tel: 0531-88365436. 


\section{1 紫外吸收光谱}

蛋白质之所以能产生紫外吸收光谱, 其主要原 因是色氨酸(Trp)和酪氨酸(Tyr)残基的侧链基团对 光的吸收, 其次是苯丙氨酸(Phe)、组氨酸(His)、半胱 氨酸(Cys)残基的侧链基团对光的吸收, 此外还有肽 键对光的强烈吸收. 其中, Trp、Tyr、Phe 3 个残基, 由 于其生色基团的不同而有不同的紫外吸收光谱. Trp 和 Tyr 在 $280 \mathrm{~nm}$ 波长附近有一个吸收峰; Phe 在 $257 \mathrm{~nm}$ 波长附近有一个吸收峰; 肽键在 $225 \mathrm{~nm}$ 波 长附近给出一个特征吸收峰. 当向蛋白质溶液中加 人表面活性剂后, 往往导致蛋白质生色基团紫外吸 收光谱的变化 ${ }^{[31-32]}$, 据此便可了解氨基酸残基微环境 的变化, 从而推断蛋白质分子在溶液中的构象变化.

Pombo 等 ${ }^{[33]}$ 通过紫外吸收光谱研究了 $25{ }^{\circ} \mathrm{C}$ 、 $\mathrm{pH}=10.0$ 时阳离子表面活性剂 $n$-烷基 $\left(\mathrm{C}_{10} \sim \mathrm{C}_{16}\right)$ 三甲 基溴化铵诱导牛胰岛素构象的变化. 以 $275 \mathrm{~nm}$ 处的 吸光度与表面活性剂浓度绘图求得了 $n$-烷基三甲 基溴化铵-牛胰岛素复合物在水中的 Gibbs 自由能 变化 $\left(\Delta G_{\mathrm{w}}^{0}\right)$ 和疏水环境中 Gibbs 自由能变化 $\left(\Delta G_{\mathrm{hc}}^{0}\right)$. 研究表明, $\Delta G_{\mathrm{w}}^{0}$ 值为 $-(11.8 \pm 1.8) \mathrm{kJ} \cdot \mathrm{mol}^{-1}$, 该值与烷 基链的长度无关并且与 $n$-烷基 $\left(\mathrm{C}_{10} \sim \mathrm{C}_{16}\right)$ 硫酸盐-牛胰 岛素复合物的 $\Delta G_{\mathrm{w}}^{0}$ 数值 ${ }^{[34}$ 接近. 由此推断, 两类表 面活性剂诱导牛胰岛素构象变化的机理类似; 当链 长从 $\mathrm{C}_{10}$ 增加到 $\mathrm{C}_{16}$ 时, $\Delta G_{\mathrm{hc}}^{0}$ 从 $-88 \mathrm{~kJ} \cdot \mathrm{mol}^{-1}$ 降低 到- $100 \mathrm{~kJ} \cdot \mathrm{mol}^{-1}$. 而从 $\Delta G_{\mathrm{hc}}^{0}$ 对 $n$-烷基三甲基溴化铵 的烷基链长和 $n$-烷基硫酸盐的烷基链长作图所得 的两条曲线基本重合可知, $\Delta G_{\mathrm{hc}}^{0}$ 与表面活性剂头基 的性质无关. 通过 $\Delta G_{\mathrm{hc}}^{0}$ 的变化趋势可以断定牛胰岛 素分子上所带的正、负电荷密度基本相同(牛胰岛素 分子含有 6 个阳离子氨基酸残基和 6 个阴离子氨基 酸残基).

\section{2 苂光光谱}

苂光光谱技术在表面活性剂与蛋白质相互作用 研究中的应用已经有大量报道 ${ }^{[35-42]}$. 通过荧光光谱不 仅可以测量胶束的聚集数, 体系的微极性和微粘度, 还可以研究表面活性剂与蛋白质的结合常数等. 常 见的方法有内源苂光、外源荧光和非辐射能量转移等.

\section{1 内源荧光}

蛋白质分子中的 Trp、Tyr 和 Phe 残基能够吸收 $270 \sim 300 \mathrm{~nm}$ 的紫外光而发出紫外菼光. Trp、Tyr 和 Phe 由于其侧链生色基团不同而产生不同的苂光光 谱, 其苂光峰 $\left(\lambda_{\text {max }}\right)$ 分别位于 $348 、 303$ 和 $282 \mathrm{~nm}$, 其
中, $\operatorname{Trp}$ 的苂光强度最大, Phe 的荧光强度最小. 因此 蛋白质的内源苂光主要是由 Trp 和 Tyr 残基所发 射. 当蛋白质溶液中加人表面活性剂时, 表面活性剂 与蛋白质之间的相互作用会导致蛋白质苂光的猝 灭, 利用此猝灭现象可以确定蛋白质的苂光猝灭机 理以及它们的结合常数等.

苂光猝灭作用可分为动态猝灭和静态猝灭作 用, 可分别用动态猝灭常数 $K_{\mathrm{SV}}$ (即 Stern-Volmer 猝 灭常数) 和静态猝灭结合常数 $K_{\mathrm{LB}}$ 来描述苂光物质 分子与猝灭剂分子之间相互作用的程度和猝灭作用 的性质. Lissi 等 ${ }^{[43}$ 通过内源苂光的测定研究了牛血 清白蛋白(BSA)和烷基吡啶盐的相互作用, 得到了 烷基吡啶盐碳链长度对 BSA 内源苂光猝灭的影响 规律. 八烷基吡啶盐对 BSA 的苂光猝灭严格遵循动 态猝灭方程 $F_{0} / F=1+K_{\mathrm{q}} \tau_{0} c_{\mathrm{Q}}=1+K_{\mathrm{Sv}} c_{\mathrm{Q}}$ (其中 $F_{0}$ 为未加 猝灭剂时苂光分子的苂光强度, $F$ 为猝灭剂浓度为 $c_{\mathrm{Q}}$ 时苂光物质的苂光强度, $K_{\mathrm{q}}$ 为双分子猝灭过程的 速率常数, $\tau_{0}$ 为不含猝灭剂时荧光分子的平均寿命, $K_{\mathrm{SV}}$ 是 Stern-Volmer 猝灭常数, 即双分子猝灭速率常 数与单分子衰变速率常数的比率), 在水溶液中和 8 $\mathrm{mol} \cdot \mathrm{L}^{-1}$ 尿素存在时其动态猝灭常数 $K_{\mathrm{SV}}$ 分别为 310 和 $280 \mathrm{~L} \cdot \mathrm{mol}^{-1}$. 十二烷基吡啶盐和十六烷基吡啶盐 是 BSA 的有效猝灭剂, 其动态猝灭曲线向上弯曲, 说明体系同时存在动态和静态猝灭. 由烷基吡啶盐 在 BSA 上的结合常数变化推断, 它们之间的相互作 用主要是疏水力所致, 静电相互作用是次要的.

我们 ${ }^{[44]}$ 通过 BSA 内源菼光测定研究了其与非 离子表面活性剂吐温-20(Tween-20)之间的相互作 用. 发现 BSA 的苂光强度随 Tween-20 浓度的增大 而降低, 而且最大发射峰波长发生蓝移, 表明 Tween-20 与 BSA 之间发生了相互作用. 结合文献 [45]求算了在不同的 $I_{0} I I$ 值 $\left(I_{0}\right.$ 为 BSA 的苂光强度, $I$ 为含有不同浓度 Tween-20 时 BSA 的苂光强度)时, Tween-20 与 BSA 的拟结合常数 (pseude-binding constant) 以及与 $1 \mathrm{~mol} \mathrm{BSA}$ 结合的 Tween-20 分子 的平均数目 $(n)$.

\section{2 外源荧光}

对于蛋白质的研究不仅可以利用其内源苂光, 而且可以通过外源苂光性质研究获得更多关于蛋白 质分子的信息. 对蛋白质-表面活性剂体系而言, 最常 用的苂光探针有萠 (Py)、1-苯胺基萘-8-磺酸(ANS)、 2-对-甲苯胺基菜-6-磺酸 (TNS)、1-( $N$-二甲基胺)-菜5 -磺酸(DNS)等, 这些苂光探针在水溶液中的量子产 


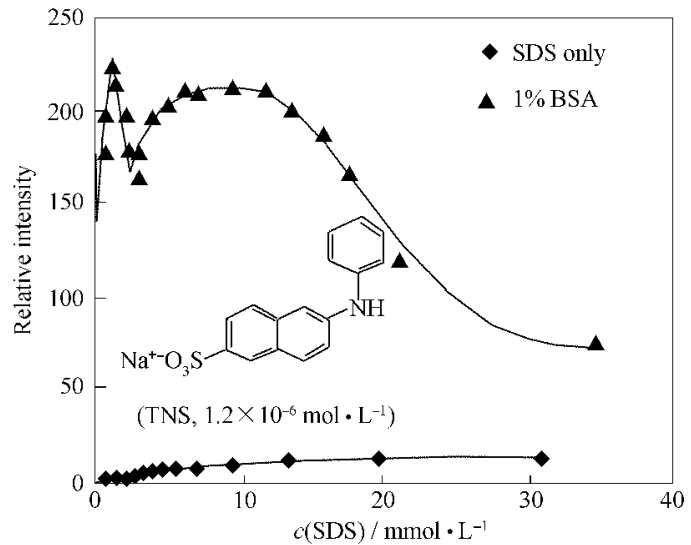

图 1 含与不含 BSA 时 TNS 的荧光强度随 SDS 浓度 的变化 ${ }^{[4]}$

Fig.1 Fluorescence intensity of TNS in the presence and absence of BSA as a function of SDS concentration ${ }^{[47]}$

BSA: bovine serum albumin; SDS: sodium dodecyl sulphate; TNS :2-p-toluidinylnaphthalenesulfonate

率很低, 但在非极性溶剂中, 其量子产率明显增大, 且苂光峰蓝移.

\subsection{1 稳态荧光}

在蛋白质-表面活性剂混合体系的稳态荧光测 量中, 以 $\mathrm{Py}$ 为探针可获得以下信息 ${ }^{[46]}$ : (1) 以 $\mathrm{Py}$ 的 第三峰 $(384 \mathrm{~nm})$ 与第一峰 $(373 \mathrm{~nm})$ 菼光强度之比 $\left(I_{3} /\right.$ $\left.I_{1}\right)$ 对表面活性剂浓度作图, 可求得蛋白质与表面活 性剂相互作用的临界聚集浓度(cac); 同时得到 Py 分子所处微环境的极性强弱 $\left(I_{3} / I_{1}\right.$ 越小, 微环境的极 性越强); (2) 以 Py 为探针, 苯甲酮为猝灭剂, 可求得 表面活性剂在混合体系中的聚集数; (3) 通过 Py 的 激基缔合物与单体苂光强度之比 $\left(I_{\mathrm{d}} / I_{\mathrm{m}}\right)$ 可推断出蛋 白质-表面活性剂聚集体的微粘度及表面活性剂聚 集体尺寸的变化.

Turro 等 ${ }^{[47}$ 利用苂光光谱详细研究了十二烷基 硫酸钠 (SDS)/BSA 体系. 采用的荧光探针是 Py 和 TNS. 与 Py 不同的是, TNS 是带有一个阴离子磺酸基 的亲水性探针, 它可与 SDS 分子的亲水基团 $-\mathrm{OSO}_{3}^{-}$ 发生竞争作用而结合到蛋白质的阳离子部位. TNS 在水溶液中的苂光强度很低, 在 SDS 胶束溶液中其 苂光强度仍然很弱, 这表明 TNS 没有在 SDS 胶束 中浓集(如图 1 所示). 当 TNS 加人到含有 $1 \%(w) B S A$ 的 SDS 溶液中, 探针的菼光强度显著增加, 同时也 发现 Py 的 $I_{3} / I_{1}$ 也有类似的变化趋势(图 2). 由图 1 可看出, 当 SDS 浓度较低时, TNS 苂光强度增加得 比较快, 表明探针周围疏水性增强, 这归因于 SDS

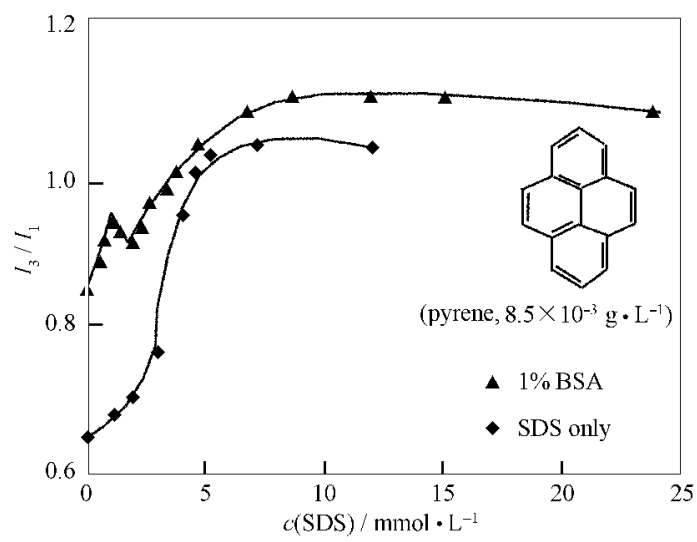

图 2 含与不含 BSA 时萠的 $I_{3} / I_{1}$ 值随 SDS 浓度的变 化 ${ }^{[47]}$

Fig.2 $I_{3} / I_{1}$ ratio of pyrene in the presence and absence of BSA as a function of SDS concentration $^{[4]}$

和 TNS 在 BSA 疏水微区附近协同与 BSA 相结合. 随着 SDS 浓度的增加, 探针周围的疏水性降低, 表 明 SDS 与 BSA 的结合占据了部分 TNS 与 BSA 结 合的部位, 导致部分 TNS 分子被释放到极性较强的 环境中. 当 $\mathrm{SDS}$ 的浓度大于 $2.7 \times 10^{-3} \mathrm{~mol} \cdot \mathrm{L}^{-1}$ 时, TNS 苂光强度又突然增强, 说明 SDS 与 BSA 结合 导致蛋白质链伸展, 所以蛋白质的疏水微区数目显 著增加. 继续增加 SDS 浓度至 $1.0 \times 10^{-2} \mathrm{~mol} \cdot \mathrm{L}^{-1}$ 时, 部分与蛋白质结合的 TNS 被 SDS 所取代, 致使 TNS 又进人水相中, 所以其荧光强度又趋于降低.

\section{2 .2 时间分辨荧光}

时间分辨苂光光谱是依据待测组分荧光衰减特 性的差异而进行选择性测定, 不仅可消除瑞利和拉 曼散射的干扰, 还可以根据苂光寿命的差异对苂光 光谱重叠组分进行测定, 为复杂体系的苂光测量提 供了选择性基础. 该方法的出现为蛋白质-表面活性 剂混合体系的研究开辟了新的途径 ${ }^{[47-49]}$.

Vasilescu 等 ${ }^{[50}$ 以 $\mathrm{Py}$ 为探针, 苯甲酮为猝灭剂, 采用稳态和时间分辨苂光光谱研究了非离子表面活 性剂八聚乙二醇十二烷基醚(octaxyethylene glycol $n$-dodecyl ether, $\mathrm{C}_{12} \mathrm{E}_{8}$ ) 与 BSA 混合体系的聚集行为. 结果表明, 采用稳态和时间分辨菼光光谱测得的聚 集数基本接近. 当 $\mathrm{C}_{12} \mathrm{E}_{8}$ 的浓度为 $6 \mathrm{mmol} \cdot \mathrm{L}^{-1}$ 时, 不 含 BSA 时, $\mathrm{C}_{12} \mathrm{E}_{8}$ 胶束的聚集数为 77 ; 若含有 $1 \%(w)$ $\mathrm{BSA}$, 其聚集数降为 35 . 但当 $\mathrm{C}_{12} \mathrm{E}_{8}$ 浓度高达 40 $\mathrm{mmol} \cdot \mathrm{L}^{-1}$ 时, 含与不含 $1 \% \mathrm{BSA}$ 时 $\mathrm{C}_{12} \mathrm{E}_{8}$ 胶束的聚 集数差别较小. 


\section{3 共振光散射光谱}

共振光散射又叫共振瑞利散射(resonance light scattering, RLS), 它是指在普通的荧光分光光度计 上选择合适的激发和发射通带宽度, 采用相等的激 发和发射波长同时扫描激发和发射单色器所得的 同步苂光光谱(即 $\Delta \lambda=0$ ). 应用该技术已成功地建立 了高灵敏度的测定蛋白质的分析方法, 为研究表面 活性剂和蛋白质等生物大分子的相互作用提供了 一个重要的方法 ${ }^{[51-53]}$. Yang 等 ${ }^{[541}$ 采用共振光散射研 究了 SDS 和十二烷基苯磺酸钠(SDBS)与 BSA 的相 互作用, 据此建立了测定痕量蛋白质的方法. 实验 表明, 在相同条件下, SDBS-BSA 混合体系的 RLS 强度要远比 SDS-BSA 混合体系的大; 并且 RLS 强 度与 BSA 的浓度成正比. 在 SDBS-BSA 混合体系 中, BSA 的线性范围为 $7.5 \times 10^{-9} \sim 1.5 \times 10^{-5} \mathrm{~g} \cdot \mathrm{mL}^{-1}$, 检 测限为 $1.8 \mu \mathrm{g} \cdot \mathrm{L}^{-1}$. 并用所拟方法测定了合成样品 中 BSA 的含量, 相对标准偏差小于 $3.6 \%$.

\section{4 荧光共振能量转移}

1948 年, Forster 提出了苂光共振能量转移理论, 它是能量给体分子(donor)和受体分子(acceptor)偶 极-偶极相互作用的结果. 根据 Forster 能量转移理 论 ${ }^{[5]}$, 当 2 种化合物发生苂光共振能量转移时必须 满足 3 个条件:(1) 能量给体分子发苂光;(2) 能量给 体分子的苂光发射光谱与能量受体分子的吸收光 谱有足够重叠; (3) 能量给体分子与能量受体分子足 够接近, 最大距离不超过 $7 \mathrm{~nm}$. 能量转移效率 $(E)$ 与 给体和受体间作用距离 $(R)$ 之间的关系可用下式表 示:

$$
E=R_{0}{ }^{6} /\left(R_{0}{ }^{6}+R^{6}\right)
$$

其中, $R_{0}$ 是能量转移效率为 $50 \%$ 时的给体-受体之 间的距离.

De 等 ${ }^{[99}$ 根据能量转移理论研究了 SDS、十六烷 基三甲基溴化铵(CTAB)和非离子表面活性剂 Triton X-100 对 BSA 中 Trp 给体与探针 ANS 受体之间能 量转移效率的影响. 研究表明, 表面活性剂存在时 Trp 与 ANS 之间的能量转移效率降低, 作用距离增 加. 由于三种表面活性剂都能与 BSA 发生相互作 用, 并且均能有效地让给体和受体暴露在溶剂中, 因此增加了给体和受体间的作用距离, 降低了能量 转移的可能性. 在这三种蛋白质/表面活性剂混合体 系中, 能量转移的速率常数的数量级均为 $10^{8} \mathrm{~s}^{-1}$, 其 顺序依次为 $\mathrm{BSA} /$ Triton X-100 $>\mathrm{BSA} / \mathrm{CTAB}>\mathrm{BSA} /$ SDS.

\section{3 圆二色光谱法}

蛋白质在溶液中存在多种二级结构, 如 $\alpha$-螺 旋、 $\beta$-折叠、 $\beta$-转角以及无规卷曲等. 加人表面活性 剂后, 由于表面活性剂与蛋白质的相互作用, 往往 会使蛋白质的结构发生一些变化, 如折叠以及去折 叠等. 通常圆二色(circular dichroism, CD)光谱法可 用于研究表面活性剂诱导蛋白质二级结构的变化.

蛋白质的 $\mathrm{CD}$ 光谱一般分为两个波长范围, 即 $178 \sim 250 \mathrm{~nm}$ 为远紫外区 $\mathrm{CD}$ 光谱, 250 320 nm 为 近紫外区 $\mathrm{CD}$ 光谱. 远紫外区是肽键的吸收峰范围, 反映了主链构象. 在远紫外区, 一般天然蛋白质的 $\mathrm{CD}$ 光谱包含一个正峰(在 $190 \mathrm{~nm}$ 波长左右)和一个 负槽(在 205 235 nm 波长范围), 其中, 负槽的形状 与主链构象密切相关. 如图 3 所示 ${ }^{[56]}, \alpha$-螺旋给出 209 和 $222 \mathrm{~nm}$ 左右的两个负槽, 称为双槽曲线; $\beta$-折叠 给出 $215 \mathrm{~nm}$ 左右的负槽. 近紫外区主要与侧链生 色团有关, 近紫外 $\mathrm{CD}$ 光谱可灵敏地反映出芳香氨 基酸残基以及二硫键的微环境变化.

Ghosh 等 ${ }^{[57}$ 采用远紫外区 $\mathrm{CD}$ 光谱研究了胰蛋 白酶与 SDS 的相互作用. 图 4 示出了 SDS 对胰蛋 白酶 $\mathrm{CD}$ 光谱的影响. 可见当胰蛋白酶的浓度较高 时, 曲线 $G 、 H$ 和 $I$ 在 220 222 nm 出现一个负槽, 表 明 $\alpha$-螺旋含量在逐渐增加. 固定胰蛋白酶的浓度, 改变 SDS 的浓度考察混合体系 $\mathrm{CD}$ 光谱(图略)的变 化时发现, 当 $\operatorname{SDS}$ 浓度为 $0.003 \mathrm{~mol} \cdot \mathrm{L}^{-1}$ 时, 肤蛋白 酶的 $\alpha$-螺旋含量为 $28 \%$, 比无 SDS 时的 $\alpha$-螺旋含 量 $(60 \%)$ 低, $\beta$-折叠和无规卷曲的含量却比无 SDS 时高. SDS 浓度较高时, $\alpha$-螺旋含量达到 $100 \%$ 并且 形成了胰蛋白酶-SDS 聚集体. 胰蛋白酶-SDS 聚集 体间强烈的静电斥力使得蛋白质分子伸展并且引 起蛋白质变性.

正因为 $\mathrm{CD}$ 光谱对于蛋白质的二级结构十分敏

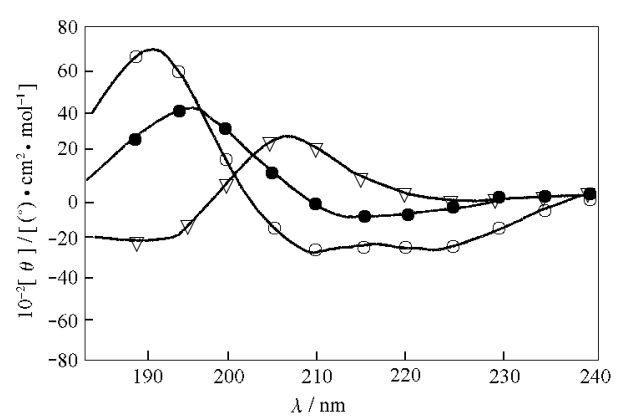

图 3 多肽的 CD 光谱 ${ }^{[56]}$

Fig.3 CD spectra of polypeptides ${ }^{[5]}$

-) $\beta$-sheet, $\nabla$ ) $\beta$-turn, O) $\alpha$-helix 


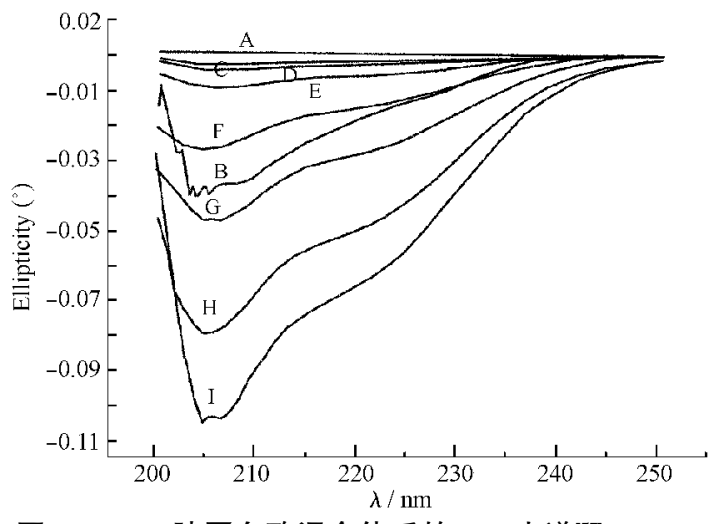

图 4 SDS-胰蛋白酶混合体系的 CD 光谱 ${ }^{[57]}$

Fig.4 CD spectra of SDS-trypsin mixtures ${ }^{[5]}$

A: $0.1 \mathrm{~mol} \cdot \mathrm{L}^{-1} \mathrm{SDS}, \mathrm{B}: 0.1 \mathrm{~g} \cdot \mathrm{L}^{-1}$ trypsin, $\mathrm{C} \sim \mathrm{I}$ : SDS $(0.1$ $\mathrm{mol} \cdot \mathrm{L}^{-1}$ )-trypsin (the concentration of trypsin is 0.001 , $0.005,0.01,0.03,0.05,0.08,0.1 \mathrm{~g} \cdot \mathrm{L}^{-1}$, respectively)

感, 所以 $\mathrm{CD}$ 光谱技术对于研究溶液中蛋白质的二 级结构及结构变化等信息具有极大的理论与实际意 $义^{[5-600]}$. 随着 $\mathrm{CD}$ 光谱技术的进一步发展, 它必将在 蛋白质与表面活性剂相互作用的研究领域中发挥重 要的作用.

\section{4 电子自旋共振光谱}

电子自旋共振(electron spin resonance, ESR), 又 叫电子顺磁共振, 它是一种微波吸收光谱技术, 可用 来研究含有未成对电子的顺磁性物质. 使用自旋探 针可把抗磁性物质体系转变成顺磁性体系, 因此自 旋探针的使用扩大了 ESR 方法的应用范围. 由于 ESR 方法具有直接检测单电子, 灵敏度高, 检测后 样品不受破坏和对化学反应无干扰等优点, 近年来 已在生物化学和胶体化学等领域中获得了广泛的应 用 ${ }^{[6-664]}$.

最常用的自旋探针是氮氧自由基, 它能以简单 的在环境中分溶的方式反映出体系内不同分子的微 环境, 因而可从分子水平上了解体系的变化, 而且可 由 ESR 光谱求得超精细分裂常数 $\left(A_{\mathrm{N}}\right)$ 和旋转相关时 间 $\left(\tau_{\mathrm{c}}\right)^{[55-66]} . A_{\mathrm{N}}$ 反映了自旋探针分子所处微环境的极 性; $\tau_{\mathrm{c}}$ 的变化则反映了自旋探针分子所处微环境粘 度的变化, $\tau_{\mathrm{c}}$ 值越大, 说明微环境粘度越大. $\tau_{\mathrm{c}}$ 可从 下面的公式求得：

$$
\tau_{\mathrm{c}}=6.5 \times 10^{-10} \times \Delta H_{0}\left[\left(\frac{h_{0}}{h_{-1}}\right)^{1 / 2}+\left(\frac{h_{0}}{h_{+1}}\right)^{1 / 2}-2\right]
$$

式中 $\Delta H_{0}$ 为中央峰的线宽; $h_{0} 、 h_{-1}$ 和 $h_{+1}$ 分别是中央 峰、高场峰和低场峰的峰高.

Turro 等 ${ }^{[47]}$ 以 5 -氮氧自由基硬脂酸 (5-doxyl

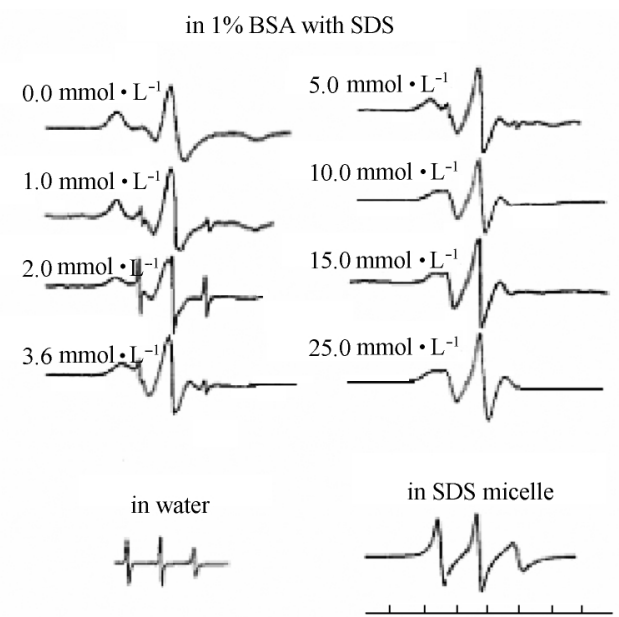

图 5 5-氮氧自由基硬脂酸在 BSA-SDS 溶液中的 ESR 光谱 ${ }^{[47]}$

Fig.5 ESR spectra of 5-doxyl stearic acid in BSA solution as a function of SDS concentration ${ }^{[4]}$

5-doxyl stearic acid concentration : $1 \times 10^{-4} \mathrm{~mol} \cdot \mathrm{L}^{-1}$

stearic acid, 5-DSA)为探针采用 ESR 光谱研究了BSA 和 SDS 混合体系微环境的性质(图 5). 5-DSA 在水中 和 SDS 胶束中的电子自旋光谱都显示出三个峰, 且 在 SDS 胶束中的三个峰线型比在水介质中要宽. 对 于 BSA-SDS 混合体系而言, 当 SDS 浓度增加到 $1.0 \sim 2.0 \mathrm{mmol} \cdot \mathrm{L}^{-1}$ 时, 探针分子探测到体系处于粘度 较低的类水环境中(三个峰比较尖), 说明探针分子 被 SDS 分子替代进人到极性更强、更易流动的区域 中, 这种现象与苂光数据得到的信息是一致的; 当 SDS 浓度为 $3.6 \mathrm{mmol} \cdot \mathrm{L}^{-1}$ 时, 被表面活性剂替代的 探针又重新增溶到疏水区域; 继续增大 SDS 浓度至 $25.0 \mathrm{mmol} \cdot \mathrm{L}^{-1}$ 时, 探针的运动要比在无 $\mathrm{BSA}$ 溶液 中的运动受到更多的约束.

\section{5 结束语}

通过以上的介绍可见, 借助于光谱技术可以研 究蛋白质结构与功能的关系以及蛋白质与表面活性 剂之间的作用机理等. 紫外吸收光谱、苂光光谱、圆 二色光谱和电子自旋共振光谱可提供相同且互为补 充的信息. 例如, 利用荧光光谱和电子自旋共振光谱 均可研究蛋白质-表面活性剂混合体系的微环境和 微粘度, 前者还可以测量胶束的聚集数及尺寸大小. 可以相信, 光谱法必将在蛋白质-表面活性剂混合体 系的研究中发挥越来越重要的作用. 但是随着科学 研究的不断发展与深人, 单一的光谱技术不能满足 蛋白质-表面活性剂混合体系物理化学性能与结构 
研究的需求, 而应该和其它技术结合起来使用, 获得 更多的信息, 以便更深人地理解它们相互作用的本 质. 同时, 新的蛋白质-表面活性剂混合体系的光谱 研究方法的不断涌现, 必将推动生命科学和胶体科 学的向前迈进.

\section{References}

1 Lucassen-Reynders, E. H. Physical chemistry of surfactant action (Surfactant science series Vol. 11). New York: Marcel Dekker Inc., $1981: 131$

2 Goddard, E. D. ; Ananthapadmanabhan, K. P. Interaction of surfactants with polymers and protein. New York: CRC Press, $1993: 320$

3 Kwak, J. C. T. Polymer-surfactant systems. New York: Marcel Dekker Inc., 1998: 267

4 Nielsen, A. D.; Borch, K.; Westh, P. Biochim. Biophys. Acta, 2000, 1479(1-2): 321

5 Otzen, D. E. Biophys. J., 2002, 83(4): 2219

6 Fainerman, V. B.; Zholob, S. A.; Leser, M. E.; Michel, M.; Miller, R. J. Colloid Interface Sci., 2004, 274: 496

7 Velev, O. D.; Pan, Y. H.; Kaler, E. W.; Lenhoff, A. M. Cryst. Growth Des., 2005, 5(1): 351

8 Fainerman, V. B.; Zholob, S. A.; Leser, M. E.; Michel, M.; Miller, R. J. Phys. Chem. B, 2004, 108: 16780

9 Gharibi, H.; Javadian, S.; Hashemianzadeh, M. Colloid Surf. A, 2004, 232: 77

10 Dickinson, E. Colloid Surf. B, 1999, 15: 161

11 Miller, R.; Fainerman, V. B.; Leser, M. E.; Michel, M. Curr Opin. Colloid Interface Sci., 2004, 9: 350

12 Miller, R.; Fainerman, V. B.; Leser, M. E.; Michel, M. Colloid Surf. A, 2004, 233 : 39

13 Giancola, C.; Sena, C. D.; Fessas, D.; Graziano, G.; Barone, G. Int. J. Biol. Macromol, 1997, 20(3): 193

14 Tribout, M.; Paredes, S.; González-Mañas, J. M.; Goñi, F. M. J. Biochem. Biophys. Methods, 1991, 22(2): 129

15 Sarmiento, F.; Ruso, J. M.; Prieto, G.; Mosquera, V. Langmuir 1998, 14: 5725

16 Stenstam, A.; Montalvo, G.; Grillo, I.; Gradzielski, M. J. Phys. Chem. B, 2003, 107: 12331

17 Stenstam, A.; Topgaard, D.; Wennerstrolm, H. J. Phys. Chem. B 2003, 107: 7987

18 Mackie, A. R.; Gunning, A. P.; Ridout, M. J.; Wilde, P. J.; Patino, J. R. Biomacromolecules, 2001, 2: 1001
19 Gunning, P. A.; Mackie, A. R.; Gunning, A. P.; Woodward, N. C.; Wilde, P. J.; Morris, V. J. Biomacromolecules, 2004, 5: 984

20 Green, R. J.; Su, T. J.; Lu, J. R.; Webster, J. R. P. J. Phys. Chem. $B, 2001,105$ : 9331

21 Valstar, A.; Almgren, M. ; Brown, W. Langmuir, 2000, 16: 922

22 Ruso, J. M.; Deo, N.; Somasundaran, P. Langmuir, 2004, 20 : 8988

23 Zhang, H. J.; Bi, Z. C.; Cui, G. C.; Li, J. B. High Technology Communication, 2000, 8: 22 [张恒建, 毕只初, 崔光晨, 李峻柏. 高技术通讯 (Gaojishu Tongxun), 2000, 8: 22]

24 Liu, J.; Xu, G. Y.; Liu, J.; Yu, L.; Zhang, H. L.; Yu, X. F. Acta Phys.-Chim. Sin., 2005, 21(8): 862 [刘 静, 徐桂英, 刘 军, 于 丽, 张洪林, 于秀芳. 物理化学学报(Wuli Huaxue Xuebao), 2005, 21(8): 862]

25 Liu, J.; Xu, G. Y.; Liu, J.; Xin, X.; Luan, Y. X. J. Disper. Sci. Technol., 2005, 26: 1

26 Wei, Q.; Wu, D.; Du, B.; Zhang, H.; Ou, Q. Y. Chinese J. Chem., 2004, 22(7): 714

27 Wei, Q.; Wu, D.; Du, B.; Li, Y.; Duan, C. H. Spectrochim. Acta Part A, 2005, (in press)

28 Wei, Q.; Wu, D.; Du, B.; Li, Y.; Wang, J. H. Anal. Sci., (Accepted)

29 Wei, Q.; Wu, D.; Du, B.; Ou, Q. Y. Chinese Chem. Lett., 2004, 15(6): 667

30 Liu, J.; Xu, G. Y.; Yuan, S. L.; Jiang, P. J. Disper. Sci. Technol., 2003, 24(6) : 779

31 Ruso, J. M.; González-Pérez, A.; Prieto, G.; Sarmiento, F. Int. J. Biol. Macromol., 2003, 33: 67

32 Tao, W. S.; Li, W.; Jiang, Y. M. The basic of protein molecules (second edition). Beijing: Higher Education Press, 1995: 238 [陶 慰孙, 李 帷, 姜涌明. 蛋白质分子基础(第二版). 北京: 高等教 育出版社, 1995: 238]

33 Pombo, C.; Prieto, G. ; del Rio, J. M.; Sarmiento, F.; Jones, M. N. Int. J. Biol. Macromol., 1996, 18: 55

34 Housaindokht, M. R.; Jones, M. N.; Newall, J. F.; Prieto, G.; Sarmiento, F. J. Chem. Soc. Faraday Trans., 1993, 89: 1963

35 Ruiz, D. L. R.; Torres, A. L. C.; García, E. A.; García, M. E. D. Analyst, 1998, 123: 2257

36 Zanette, D.; Lima, C. F.; Ruzza, A. A.; Belarmino, A. T. N.; Santos, S. D. F.; Frescura, V. L. A.; Marconi, D. M. O.; Froehner, S. J. Colloids Surf. A, 1999, 147: 89

37 Moriyama, Y.; Ohta, D.; Hachiya, K.; Mitsui, Y.; Takeda, K. J. Protein Chem., 1996, 15(3): 265 
38 Gorinstein, S.; Goshev, I. ; Moncheva, S.; Zemser, M.; Weisz, M.; Caspi, A.; Libman, I.; Lerner, H. T.; Trakhtenberg, S.; Martin-Belloso, O. J. Protein Chem., 2000, 19(8): 637

39 Daban, J. R.; Samsó, M.; Bartolomé, S. Anal. Biochem.,1991, 199(2): 162

40 Lee, C. T.; Smith, K. A.; Hatton, T. A. Biochemistry, 2005, 44: 524

41 Tofani , L.; Feis, A.; Snoke, R. E.; Berti, D.; Baglioni, P.; Smulevich, G. Biophys. J., 2004, 87: 1186

42 Ji, Y.; Zhang, X. H.; Guo, R. Acta Chimica Sinica, 2004, 62(4): 345 [纪 云, 张晓红, 郭 荣. 化学学报 (Hиахие Хиеbao), 2004, 62(4): 345]

43 Diaz, X.; Abuin, E.; Lissi, E. J. Photoch. Photobio. A, 2003, 155: 157

44 Liu, J. Master Dissertation. Shandong: Shandong University, 2004 [刘 静. 硕士学位论文. 山东: 山东大学, 2004]

45 Lissi, E.; Abuin, E.; Lanio, M. E.; Alvarez, C. J. Biochem. Biophys. Methods, 2002, 50: 261

46 Xu, G. Y.; Luan, Y. X.; Liu, J.; Yu, L. Acta Phys.-Chim. Sin., 2005, 21(5): 577 [徐桂英, 栾玉霞, 刘 静, 于 丽. 物理化学 学报(Wuli Huaxue Xuebao), 2005, 21(5): 577]

47 Turro, N. J.; Lei, X. G.; Ananthapadmanabhan, K. P.; Aronson, M. Langmuir, 1995, 11 : 2525

48 Gelamo, E. L.; Silva, C. H. T. P.; Imasato, H.; Tabak, M. Biochim. Biophys. Acta, 2002, 1594: 84

49 De, S.; Girigoswami, A.; Das, S. J. Colloid Interf. Sci., 2005, 285: 562

50 Vasilescu, M.; Angelescu, D. Langmuir, 1999, 15: 2635

51 Jiang, B.; Hu, Q. H. Chinese J. Anal. Lab., 2002, 21(3): 27 [江 波. 胡庆红. 分析实验室(Fenxi Shiyanshi), 2002, 21(3): 27]
52 Jiang, B.; Hu, Q. H. Journal of Guanxi Normal University, 2002, 20(2): 60 [江 波. 胡庆红. 广西师范大学学报(Guangxi Shifan Dахие Хиеьао), 2002, 20(2): 60]

53 Hu, Q. H.; Jiang, B. Chin. J. Anal. Chem., 2003, 31(9): 1123 [胡庆红. 江 波. 分析化学(Fenxi Hиахиe), 2003, 31(9): 1123]

54 Liu, R. T.; Yang, J. H.; Sun, C. X.; Wu, X. ; Li, L.; Li, Z. M. Anal. Bioanal. Chem., 2003, 377: 375

55 Clegg, R. M. Curr. Opin. Biotech., 1995, 6: 103

56 Brahms, S.; Brahms, J. J. Mol. Biol., 1980, 138(2): 149

57 Ghosh, S.; Banerjee, A. Biomacromolecules, 2002, 3: 9

58 Moriyama, Y.; Takeda, K. Langmuir, 2005, 21(12): 5524

59 Sun, C. X.; Yang, J. H.; Wu, X.; Huang, X. R.; Wang, F.; Liu, S. F. Biophys. J., 2005, 88(5): 3518

60 Deep, S.; Ahluwalia, J. C. Phys. Chem. Chem. Phys., 2001, 3 : 4583

61 Fukuda, H., Goto, A.; Yoshioka, H.; Goto, R.; Morigaki, K.; Walde, P. Langmuir, 2001, 17: 4223

62 Hai, M. T.; Han, B. X.; Yan, H. K. J. Phys. Chem. B, 2001, 105 : 4824

63 Gelamo, E. L.; Itri, R.; Alonso, A.; Silva da, J. V.; Tabak, M. J. Colloid Interf. Sci., 2004, 277 : 471

64 Middleton, H.; English, R. J.; Williams, P. A.; Broze, G. Langmuir, 2005, 21(11): 5174

65 Shi, S.; Hao, J. C.; Lu, R. H.; Wang, T. T.; Wang, H. Q. China Surfactant Detergent \& Cosmetics, 1997, 1: 53 [石 硕, 郝京 诚, 鲁润华, 王淘淘, 汪汉卿. 日用化学工业 (Riyong Huaxue Gongye), 1997, 1 : 53]

66 Dong, S. L.; Xu, G. Y. Acta Chimica Sinica, 2004, 62(7): 674 [董姝丽, 徐桂英. 化学学报(Hиахие Хиеbao), 2004, 62(7): 674] 